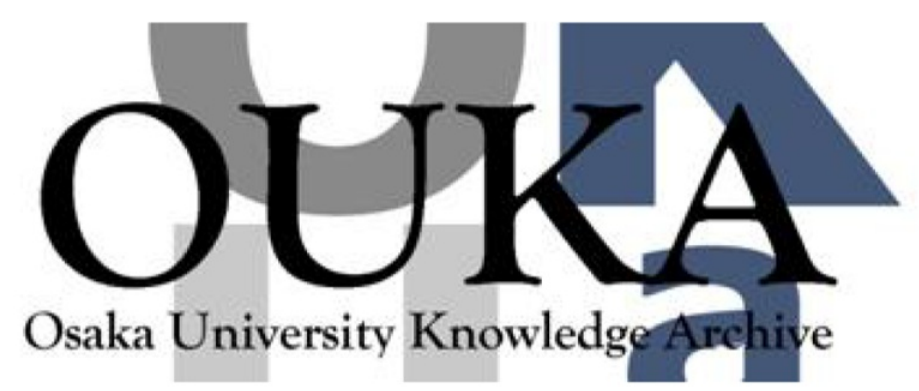

\begin{tabular}{|c|l|}
\hline Title & $\begin{array}{l}\text { Preparation of organic nanoscrews from simple } \\
\text { porphyrin derivatives }\end{array}$ \\
\hline Author(s) & $\begin{array}{l}\text { Ozawa, Hiroaki; Tanaka, Hirofumi; Kawao, } \\
\text { Masahiro et al. }\end{array}$ \\
\hline Citation & Chemical Communications. 47 p. 7411-p. 7413 \\
\hline Issue Date & 2009 \\
\hline oaire:version VoR \\
\hline URL & https://hdl. handle. net/11094/3120 \\
\hline rights & \\
\hline Note & \\
\hline
\end{tabular}

Osaka University Knowledge Archive : OUKA

https://ir. Library. osaka-u. ac. jp/

Osaka University 


\title{
Preparation of organic nanoscrews from simple porphyrin derivatives $\dagger$
}

\author{
Hiroaki Ozawa, $\ddagger^{* a}$ Hirofumi Tanaka, ${ }^{b}$ Masahiro Kawao, ${ }^{c}$ Shigeyasu Uno ${ }^{a}$ and \\ Kazuo Nakazato $* a$
}

Received (in Cambridge, UK) 3rd August 2009, Accepted 13th October 2009

First published as an Advance Article on the web 28th October 2009

DOI: $10.1039 /$ b915832d

Twisted supramolecular assemblies "nanoscrews", were prepared by using simple porphyrin derivatives and acetonitrile solvent. The electrical properties of the assemblies were measured by using microgap electrodes. The nanoscrew had conductivity and showed photo-current. By changing the solvent, the pitch of the screws and their aggregation shapes could be controlled.

Supramolecular assemblies consisting of $\pi$-conjugated molecules in the micro/nanoscale are attracting considerable interest because of their potential applications in molecular and supramolecular electronics. ${ }^{1,2}$ Recently, many studies on supramolecular assemblies have been reported, and various sophisticated structures such as spheres, discs, sheets, rods, and wheels have been developed using these assemblies. ${ }^{3}$ In particular, twisted or coiled structures are promising candidates for the investigation of electromagnetic induction in nanoscale devices. ${ }^{4}$ The preparation of twisted supramolecular assemblies, formed from hexaperihexabenzocoronene derivatives, have been reported, and the twisting direction of one-dimensional assemblies has been successfully controlled. ${ }^{5}$

Porphyrin derivatives are one of the most popular materials and known as a powerful tool for the construction of supramolecular assemblies. ${ }^{6}$ The $\pi-\pi$ stacking interaction inherent to their $\pi$-conjugated skeletons is considered to be the major driving force in the formation of these supramolecular assemblies. Moreover, porphyrin is often used to prepare micro/nanoscale structures that can perform many of the essential light-harvesting and electron- and energy-transfer functions. ${ }^{7}$

The complex-structured molecules are generally utilized as building blocks for preparation of complex assemblies such as twisted and coiled structures. These materials are not suitable for practical use because their complicated shapes make their synthesis laborious. If twisted assemblies can be obtained using simple porhyrin derivatives, the scope of application of

\footnotetext{
${ }^{a}$ Department of Electrical Engineering and Computer Science, Graduate School of Engineering, Nagoya University, Furo-cho, Chikusa-ku, Nagoya 464-8603, Japan.

E-mail: hiro.ozawa@mail.cstm.kyushu-u.ac.jp

${ }^{b}$ Department of Chemistry, Graduate School of Science,

Osaka University, 1-1 Machikaneyama, Toyonaka 560-0043, Japan

${ }^{c}$ Research Center for Molecular-Scale Nanoscience, Institute for

Molecular Science, 5-1 Higashiyama, Myodaiji, Okazaki 444-8787.

Japan

$\dagger$ Electronic supplementary information (ESI) available: Images taken by optical microscopy, fluorescence microscopy and SEM of supramolecular assemblies; measurement of FT-IR and reflection spectra of supramolecular assemblies; and photoresponse characteristics of supramolecular assemblies. See DOI: 10.1039/b915832d

$\ddagger$ Present address: Department of Applied Chemistry Kyushu

University, 744 Motooka, Nishi-ku, Fukuoka 819-0395, Japan.
}

these assemblies in the field of molecular or supramolecular electronics will increase considerably. We have attempted to use the simple porphyrin $\mathbf{1}$ bearing two interaction moieties to obtain twisted supramolecular assemblies (Fig. 1(a)). The porphyrin ring acts as a moiety exhibiting $\pi-\pi$ stacking interaction and the 4 alkyl groups act as moieties exhibiting van der Waals interaction. ${ }^{8}$ Additionally, the optical and electrical properties of these assemblies are measured using microgap electrodes.

5,10,15,20-tetraheptyl-21H,23H-porphyrin (1) is synthesized according to previous reports. ${ }^{9} \mathbf{1}$ is characterized by ${ }^{1} \mathrm{H}$ and ${ }^{13} \mathrm{C}$ NMR, FT-IR, and UV-Vis spectroscopy, as well as by MALDI-TOF mass spectroscopy. When $\mathbf{1}$ was dispersed in acetonitrile, a turbid purple-colored dispersion was obtained. Optical microscope observations (Fig. 1(b)) revealed the formation of dendritic aggregates. Scanning electron microscope (SEM) and transmission electron microscope (TEM) observations were carried out to clarify the detailed structures of the dendritic aggregates (Fig. 1(c) and (d)). The length of the twisted supramolecular assemblies ranged from several tens to hundreds of micrometers, and their width ranged from several hundreds to thousands of micrometers. The ratio of the number of left-handed twists to that of right-handed twists in the assemblies was estimated to be $1: 1$; however, the twisting direction in a aggregate was uniform. The twisting direction may be controlled by adding seed crystals to the solution.

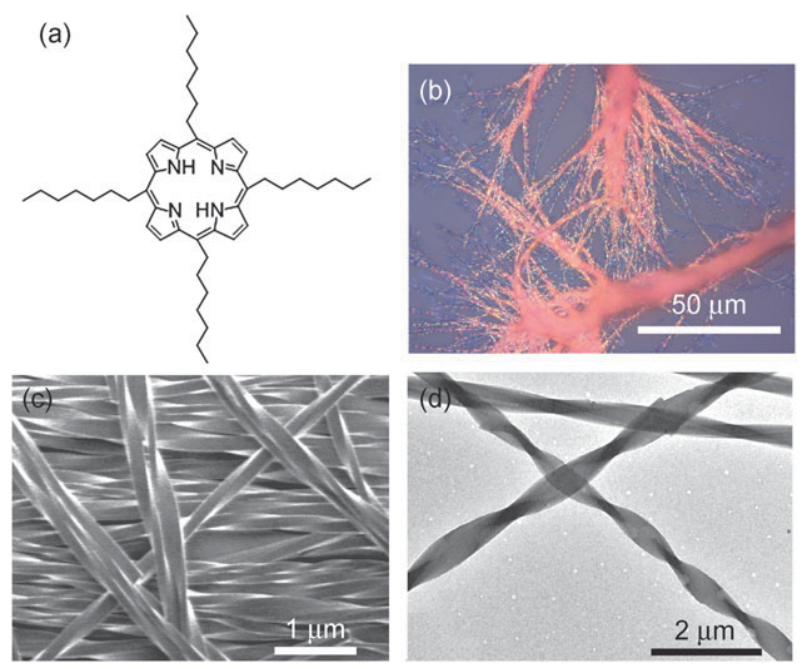

Fig. 1 (a) Structure of porphyrin 1. (b) Optical microscope image of supramolecular assemblies fabricated from porphyrin 1. (c) SEM and (d) TEM images of supramolecular assemblies. The supramolecular assemblies have a twisted structure. 
The morphological changes in the self-assembly of $\mathbf{1}$ was investigated by dissolving 1 in different solvents. The maximum possible amount of $\mathbf{1}$ was dissolved in 2-propanol, methyl lactate, and 2-(2-(2-methoxyethoxy)ethoxy)ethanol solutions $\left(0.1 \mathrm{mg} \mathrm{ml}^{-1}\right)$ at $60{ }^{\circ} \mathrm{C}$. After cooling, the solutions were cast on a glass or silicon substrate, and then the samples were observed by using optical microscopy and SEM. 1 in 2-propanol self-assembles into sheet aggregates during drying. The aggregation of the sheet structures was confirmed by optical microscope and SEM observations (Fig. S1 $\dagger$ ). The sheetshaped objects were several tens of micrometers in length and several micrometers in width. Elongated supramolecular assemblies were obtained when 2-(2-(2-methoxyethoxy)ethoxy)ethanoltri(ethyleneglycol)monomerether was used as the solvent, (Fig. S2 $\dagger$ ). Further, the supramolecular assemblies in methyl lactate and acetonitorile had twisted structures. See supporting information for images of optical microscopy and SEM of the twisted assemblies (Fig. S3†).

A scheme illustrating the formation of twisted supramolecular assemblies is shown in Fig. 2. The formation process of the twisted assemblies involved two stages. In the first stage, $\mathbf{1}$ assembled to form the core structures of the twisted assemblies. The formation of the twisted structures could be attributed to presence of defects in the packing structures of porphyrin; these defects disturb the formation of the rod structure. ${ }^{10}$ In the second stage, the core structures aggregated to form dendritic aggregates. In order to clarify these mechanisms, we carried out the following experiment. Compound $\mathbf{1}$ was dissolved in hot solvents and cast on a glass substrate. Then, the solvents were rapidly dried to disperse the precipitates formed. Optical microscopy images of the obtained supramolecular assemblies are shown in Fig. S4. $†$ In the case of acetonitrile used as the solvent, small twisted assemblies were observed on the substrate. Compared with the dendrite aggregation, the size and the number of present twisted assemblies are small. Note that rapid drying led to the formation of intermediate structures. The results confirmed the proposed formation mechanism of supramolecular assemblies.

It is well known that the formation of chiral compounds is induced by asymmetric hydrocarbons and chiral solvents. ${ }^{11}$ We have attempted to control the chiral direction during the preparation of supramolecular assembly by using methyl $(R)$-lactate or methyl $(S)$-lactate. However, obtained assemblies were racemic (Fig. S5(a) and S5(b)†). The ratio of the number of left-handed twists to the number of right-handed twists in the products formed when methyl $(R)$-lactate or methyl $(S)$-lactate is used are shown in Fig. S5(c) $\dagger$ (the amount ratio of opposite direction of the twisted structures was $52: 48)$ and

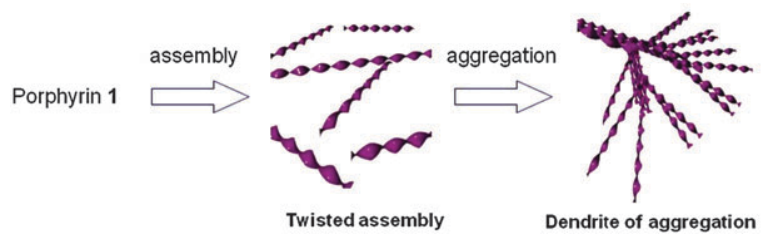

Fig. 2 Scheme illustrating the formation mechanism of a twisted assembly and dendrites of the assembly.
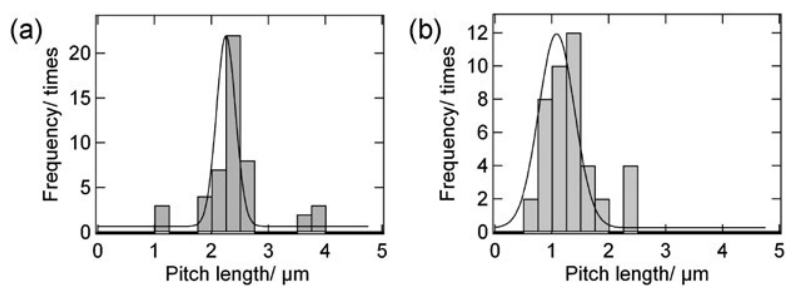

Fig. 3 Histograms of pitch length of twisted assemblies formed in (a) acetonitrile and (b) methyl $(R)$-lactate.

Fig. S5(d) $\dagger$ (the ratio was 58:42), respectively. The chiral environment have no apparent effect on the twisting direction of assemblies during their growth. Although it is difficult to control the twisting direction, the pitch length of the twisted structures can be controlled by changing the solvent. The pitch lengths of the twisted structures obtained when acetonitrile and methyl $(R)$-lactate are used as solvents are $2.25 \pm 0.23$ and $1.08 \pm 0.45 \mu \mathrm{m}$, respectively (Fig. 3), which have been confirmed from SEM images (Fig. S6†). Thus, the difference in the pitch length of the twisted structures might be influenced by solvents. The packing structures of porphyrin in assemblies must have been changed depending on the type of solvent, thereby leading out the formation of twisted structures with different pitch lengths.

We have measured the UV-Vis absorption spectra of the twisted assemblies formed on the glass substrates as shown in Fig. 4(a), in order to examine the electronic interaction of porphyrin in the structures. These spectra exhibit considerably broader and more intense absorption bands in the visible and near-infrared regions than those of compounds 1 in toluene solution. This difference in the absorption spectra can be attributed to the strong interaction between porphyrin and its nearest neighbor in the assemblies. ${ }^{12}$ The reflection spectra of the twisted assemblies formed on the glass substrates are shown in Fig. S7. $\dagger$ The characteristics of these spectra are similar to those of the UV-Vis absorption spectra. The fluorescence spectra of the aggregates formed on the glass substrates are shown in Fig. 4(b). The excitation wavelength was $410 \mathrm{~nm}$. The emission peaks of the twisted assemblies formed on the glass substrates are found to be red-shifted compared to the peak from compound $\mathbf{1}$ in toluene. These results indicate the strong interactions of porphyrins caused by the formation of assemblies. The fluorescence microscopy images of the assemblies formed on the glass substrates are shown in Fig. S8. $\dagger$ In this case, definite luminous structures formed by the aggregation of porphryin was observed.
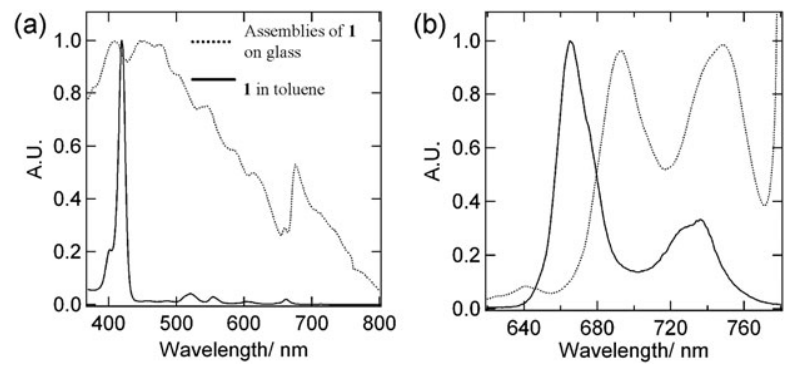

Fig. 4 (a) UV-Vis absorption spectra and (b) fluorescence spectra of twisted (dotted line) structures formed on glass substrate and compound $\mathbf{1}$ in toluene solution (solid line). 

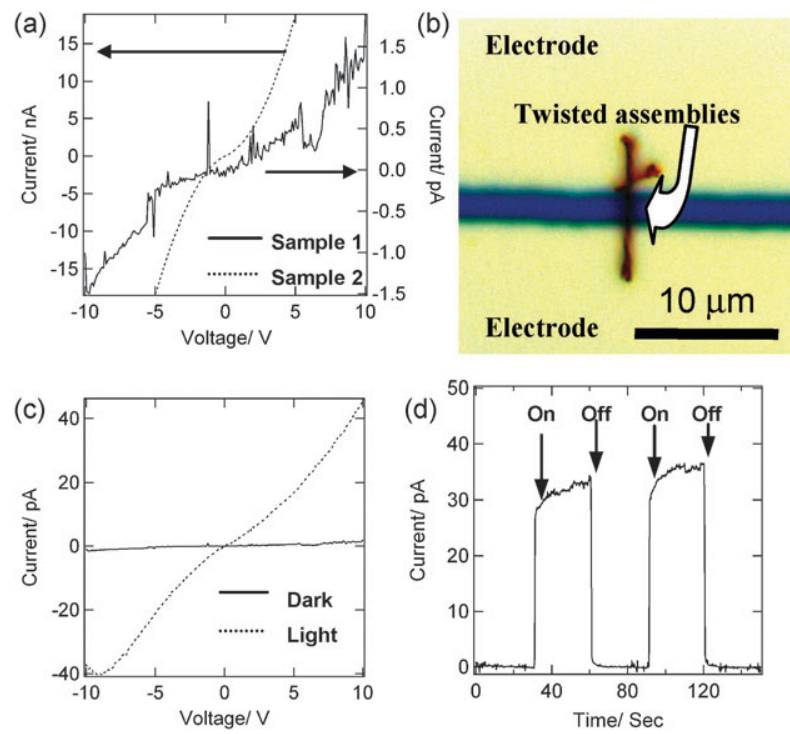

Fig. 5 (a) $I-V$ curves of twisted assemblies between $4 \mu \mathrm{m}$ gap electrodes. The arrows indicate the vertical axis corresponding to the samples. (b) Optical microscope image of twisted assemblies between electrodes. (c) $I-V$ curve of twisted assemblies between $4 \mu \mathrm{m}$ of gap electrodes with or without light irradiation. (d) Dependence of current on time at $-10 \mathrm{~V}$ when light is turned on and off periodically at room temperature.

The FT-IR spectra of the twisted assemblies are shown in Fig. S9. $\dagger$ The FT-IR spectra of the twisted assemblies formed on a $\mathrm{KCl}$ solid surface exhibit $\mathrm{CH}_{2}$ stretching vibrations at 2913 and $2846 \mathrm{~cm}^{-1}$. These bands are red-shifted as compared to those of $\mathbf{1}$ (2919 and $2850 \mathrm{~cm}^{-1}$ ). Besides, the peaks at 779 and $717 \mathrm{~cm}^{-1}$ are found to shift to 776 and $713 \mathrm{~cm}^{-1}$, respectively. Thus, the alkyl chains tend to form crystalline packing. ${ }^{13}$ These results indicate that each porphyrin molecule interacts and self-aligns, thereby forming assembly structures.

The measurements of $I-V$ characteristics of the twisted assemblies were performed with $4 \mu \mathrm{m}$ gap electrodes (Fig. 5(a)). Two similar samples were prepared (samples 1 and 2) for the measurement of the $I-V$ characteristics from -10 to $10 \mathrm{~V}$ with an interval of $0.2 \mathrm{~V}$ at room temperature. The shapes of the $I-V$ curves of the nanoscrews are similar to those of porphyrin rods or organic molecular assemblies reported previously. ${ }^{10,14}$ Photocurrent was also observed in the $I-V$ measurement. An optical microscopy image of the sample used for the measurement is shown in Fig. 5(b). The results of photocurrent measurement of this sample are shown in Fig. 5(c). The time-current scan mode with $0.2 \mathrm{~s}$ intervals is used for $150 \mathrm{~s}$ to record the photoresponse properties, and the light source was switched on and off periodically (Fig. 5(d)). When the sample is irradiated with white light, the current increases dramatically. Reproducible results are also shown in Fig. S10. $\dagger$ The mechanism might be a photoexcited electron transfer. When light is incident on the sample, electrons are photoexcited from the Highest Occupied Molecular Orbitals (HOMOs) to the Lowest Unoccupied Molecular Orbitals (LUMOs) of the porphyrin molecules. The excited electrons are then transferred to neighboring porphyrin LUMOs, thereby generating large electron diffusion. The same mechanism was observed in the case of self-assembled porphyrin nanorods. ${ }^{10}$

In conclusion, we succeeded in preparing supramolecular assemblies using simple porphyrin derivatives. The obtained assemblies had a twisted nanoscrew structure. By changing the solvent, the pitch of the screws and their aggregation shapes could be controlled. The optical and electrical properties of the nanoscrews were measured. A study on their detailed structure, control of their twist direction, and the dependence of the twist direction on conductivity measurements is underway. Detailed information on the synthetic control, alignment, and the photoresponse characteristics of supramolecular assemblies will be obtained for future applications of these assemblies to molecular electronics.

One of the authors (HO) would like to thank Prof. T. Ogawa of Osaka University for his fruitful discussion.

\section{Notes and references}

1 (a) J. M. Lehn, Angew. Chem., Int. Ed. Engl., 1988, 27, 89; (b) J. M. Lehn, Angew. Chem., Int. Ed. Engl., 1990, 29, 1304; (c) G. M. Whitesides and B. Grzybowski, Science, 2002, 295, 2418.

2 (a) A. R. Pease, J. O. Jeppesen, J. F. Stoddart, Y. Luo, C. P. Collier and J. R. Heath, Acc. Chem. Res., 2001, 34, 433; (b) Z. J. Chen, V. Stepanenko, V. Dehm, P. Prins, L. D. A. Siebbeles, J. Seibt, P. Marquetand, V. Engel and F. Wurthner, Chem.-Eur. J., 2007, 13, 436.

3 (a) Y. M. Jeon, J. Heo and C. A. Mirkin, J. Am. Chem. Soc., 2007, 129, 7480; (b) Y. B. Lim, K. S. Moon and M. Lee, J. Mater. Chem., 2008, 18, 2909; (c) J. Weiss, E. Jahnke, N. Severin, J. P. Rabe and H. Frauenrath, Nano Lett., 2008, 8, 1660; (d) S. J. Guo and E. K. Wang, Langmuir, 2008, 24, 2128.

4 B. Kobe and A. V. Kajava, Trends Biochem. Sci., 2000, 25, 509.

5 Y. Yamamoto, T. Fukushima, Y. Suna, N. Ishii, A. Saeki, S. Seki, S. Tagawa, M. Taniguchi, T. Kawai and T. Aida, Science, 2006, 314, 1761.

6 (a) T. Yamaguchi, T. Kimura, H. Matsuda and T. Aida, Angew. Chem., Int. Ed., 2004, 43, 6350; (b) Z. Wang, C. J. Medforth and J. A. Shelnutt, J. Am. Chem. Soc., 2004, 126, 15954; (c) C. M. Drain, G. Smeureanu, S. Patel, X. C. Gong, J. Garno and J. Arijeloye, New J. Chem., 2006, 30, 1834; (d) S. J. Lee, K. L. Mulfort, J. L. O'Donnell, X. B. Zuo, A. J. Goshe, P. J. Wesson, S. T. Nguyen, J. T. Hupp and D. M. Tiede, Chem. Commun., 2006, 4581.

7 (a) T. Hasobe, K. Saito, P. V. Kamat, V. Troiani, H. J. Qiu, N. Solladie, K. S. Kim, J. K. Park, D. Kim, F. D'Souza and S. Fukuzumi, J. Mater. Chem., 2007, 17, 4160; (b) T. Hasobe, H. Oki, A. S. D. Sandanayaka and H. Murata, Chem. Commun., 2008, 724.

8 (a) T. Nakanishi, W. Schmitt, T. Michinobu, D. G. Kurth and K. Ariga, Chem. Commun., 2005, 5982; (b) X. Q. Li, V. Stepanenko, Z. J. Chen, P. Prins, L. D. A. Siebbeles and F. Wurthner, Chem. Commun., 2006, 3871.

9 M. J. Crossley, P. Thordarson, J. P. Bannerman and P. J. Maynard, J. Porphyrins Phthalocyanines, 1998, 2, 511.

10 A. D. Schwab, D. E. Smith, B. Bond-Watts, D. E. Johnston, J. Hone, A. T. Johnson, J. C. de Paula and W. F. Smith, Nano Lett., 2004, 4, 1261.

11 (a) J. Aimi, A. Tsuda and T. Aida, Angew. Chem., Int. Ed., 2007, 46, 2031; (b) T. Nakanishi, K. Ariga, T. Michinobu, K. Yoshida, H. Takahashi, T. Teranishi, H. Mohwald and D. G. Kurth, Small, 2007, 3, 2019.

12 D. Kim and A. Osuka, J. Phys. Chem. A, 2003, 107, 8791.

13 O. Ohno, Y. Kaizu and H. Kobayashi, J. Chem. Phys., 1993, 99, 4128.

14 (a) K. Araki, H. Endo, H. Tanaka and T. Ogawa, Jpn. J. Appl. Phys., 2004, 43, L634; (b) A. P. H. J. Schenning and E. W. Meijer, Chem. Commun., 2005, 3245. 